\title{
Native electrospray ionization mass spectrometry reveals multiple facets of aptamer-ligand interactions: from mechanism to binding constants
}

\author{
Journal Article \\ Author(s): \\ Gülbakan, Basri; Barylyuk, Konstantin; Schneider, Petra; Pillong, Max; Schneider, Gisbert (i); Zenobi, Renato (D) \\ Publication date: \\ 2018-06-20 \\ Permanent link: \\ https://doi.org/10.3929/ethz-b-000264221
}

Rights / license:

In Copyright - Non-Commercial Use Permitted

Originally published in:

Journal of the American Chemical Society 140(24), https://doi.org/10.1021/jacs.7b13044 


\title{
Native electrospray ionization mass spectrometry reveals multiple facets of aptamer-ligand interactions: from mechanism to binding constants
}

\author{
Basri Gülbakan, ${ }^{a, b \dagger}$ Konstantin Barylyuk ${ }^{a, c \dagger}$, Petra Schneider ${ }^{a}$, Max Pillong ${ }^{a}$, Gisbert Schneider ${ }^{a}$, \\ and Renato Zenobi ${ }^{\mathrm{a}}$ \\ a.Department of Chemistry and Applied Bioscience, ETH Zürich, CH-8093 Zürich, Switzerland. \\ b. Hacettepe University Institute of Child Health, Ihsan Dogramaci Children's Hospital, Sıhhiye \\ 06100 Ankara, Turkey. \\ c. current address: Department of Biochemistry, University of Cambridge, Hopkins Building, \\ Tennis Court Road, Cambridge CB2 1QW, U.K.
}

†B G. and K.B contributed equally to this work

KEYWORDS. Oligonucleotide aptamers, native mass spectrometry, ion mobility spectrometry, binding constants

\begin{abstract}
Aptamers are oligonucleotide receptors obtained through an iterative selection process from random-sequence libraries. While many aptamers for a broad range of targets with high affinity and selectivity have been generated, a lack of high-resolution structural data and the limitations of currently available biophysical tools greatly impede understanding of the mechanisms of aptamer-ligand interactions. Here we demonstrate that an approach based on native electrospray ionization mass spectrometry (ESI-MS) can be successfully applied to characterize aptamer-ligand complexes in all details. We studied an adenosine-binding aptamer (ABA), a Largininamide-binding aptamer (LABA), and a cocaine-binding aptamer (CBA) and their noncovalent interactions with ligands by native ESI-MS and complemented these measurements by ion mobility spectrometry (IMS), isothermal titration calorimetry (ITC), and circular dichroism (CD) spectroscopy. The ligand selectivity of the aptamers and the respective complex stoichiometry could be determined by the native ESI-MS approach. The ESI-MS data can also help refining the binding model for aptamer-ligand complexes and deliver accurate aptamer-ligand binding affinities for specific and nonspecific binding events. For specific ligands we found $\mathrm{K}_{\mathrm{d} 1}=69.7 \mu \mathrm{M}$ and $\mathrm{K}_{\mathrm{d} 2}=5.3 \mu \mathrm{M}$ for ABA (two binding sites); $\mathrm{K}_{\mathrm{d} 1}=22.04 \mu \mathrm{M}$ for LABA; and $\mathrm{K}_{\mathrm{d} 1}=8.5 \mu \mathrm{M}$ for CBA.
\end{abstract}




\section{Introduction}

Oligonucleotides can be evolved in vitro into affinity probes and recognition tools called "aptamers" to bind different targets with high specificity and high selectivity.$^{1-2}$ Aptamers are single-stranded DNA or RNA molecules selected from random oligonucleotide libraries through an iterative process called Systematic Evolution of Ligands by EXponential enrichment (SELEX). Since the inception of SELEX, aptamers have been generated for a broad variety of targets, ranging from metal ions and small molecules to clinically important proteins, glycans, and even whole prokaryotic and eukaryotic cells. ${ }^{3-4}$ While the number of applications is growing rapidly, very little attention has been given to the mechanistic characterization of aptamer-target interactions. Generally, aptamers are thought to adopt a unique three-dimensional structure upon ligand binding and recognize their targets via the collective action of noncovalent interactions ${ }^{5-6}$. However, it is not known whether this binding mechanism generally holds true.

While well over thousand aptamers have been developed so far, the structures of only a handful of them have been experimentally determined, limiting our understanding of principles of the structural plasticity and binding dynamics of aptamers ${ }^{7-8}$. Recent advances in molecular dynamics (MD) simulation of oligonucleotides addressed this limitation in part and allowed studying the stability and dynamics of DNA and RNA duplexes and hairpins, elucidation of allosteric binding mechanisms, and characterizing binding of proteins, drugs, and ions to DNA. ${ }^{9-16}$ More recently, MD simulations have been applied to probe aptamer-ligand interactions ${ }^{17-25}$. However, the lack of fully resolved structures and complementary computational studies greatly impedes the progress in the rational design of new aptamers and represents a major gap in our knowledge of fundamental principles of nucleic acid interactions and binding.

Aptamer-ligand interactions have mostly been studied by methods such as fluorescence polarization/anisotropy ${ }^{26-27}$, surface plasmon resonance ${ }^{28}$, quartz crystal microbalance ${ }^{29}$, 
and capillary electrophoresis ${ }^{30}$. While these methods are useful, they require labelling or surface immobilization, which may alter the aptamer-ligand binding. Isothermal titration calorimetry (ITC), while label-free, uses high sample quantities and is therefore not practical when sample amount is an issue ${ }^{31}$. Furthermore, the information extracted from these measurements is usually limited only to the binding strength. NMR spectroscopy ${ }^{32-}$ ${ }^{33}$ and X-Ray crystallography provide structural data at the atomic level of resolution but they are quite labour-intense, which reduces their throughput and sometimes applicability ${ }^{34}$. Therefore, there is a great need for a label-free and sensitive method to study aptamer-ligand interactions, which will ideally provide both structural insight and quantitative information on binding affinity.

In the past three decades, mass spectrometry (MS) has become the most diverse and information-rich analytical technique ${ }^{35-36}$. One area where MS has become very powerful is the characterization of non-covalent biomolecular interactions and the study of higherorder structures of large biomolecular complexes. An approach termed "native electrospray ionization mass spectrometry" (native ESI-MS) has emerged as a complementary tool to NMR spectroscopy and X-Ray crystallography. Native ESI-MS preserves biomolecules and their interactions in the gas phase, and often structural insights can be obtained. ${ }^{37}$ Peak intensities in ESI mass spectra correlate with the analyte amounts in the samples, which enables determination of receptor-ligand binding constants if proper response factors and proxy ligands or proxy proteins are employed to account for imbalances in the ionization efficiencies and nonspecific binding. ${ }^{38-39}$ Compared to other biophysical methods, native ESI-MS offers several benefits: it only requires small sample volumes $(10-100 \mu \mathrm{L})$ and concentrations (low-nM to low- $\mu \mathrm{M})$, no chemical labelling is needed, and measurements are performed within a short time (5-10 s). Many different protein-ligand, protein-protein complexes, membrane proteins, 
molecular machines and large biomolecular assemblies have been studied by native ESI-MS. ${ }^{40-42}$

Since the pioneering work of Smith and coworkers to probe DNA duplex structures by $\mathrm{ESI}_{\mathrm{MS}}{ }^{43}$, ligand binding to DNA minor groove or via intercalation into base pair stacks, characterization of duplex, triplex, and quadruplex structures and folding pathways have been successfully demonstrated ${ }^{44-48}$. Cassiday and coworkers were the first to apply ESI-MS to determine the stoichiometry of aptamer-ligand complex. They showed that a 31-nucleotide RNA aptamer specifically binds to human transcription factor NF-kB p50 homodimer $^{49}$. ESI-MS studies of aptamers that specifically recognise and bind tobramycin, adenosine triphosphate (ATP), flavin mononucleotide (FMN), Largininamide, thrombin, and streptavidin have been reported ${ }^{50-52-53}$. Hydrogendeuterium exchange MS (HDX-MS) has also been used to probe aptamer-protein interactions $^{54}$.

While native ESI-MS is widely applied to study protein complexes, aptamer-ligand interactions have not been investigated by this method in a systematic way. One reason for this is the polyanionic nature of nucleic acids, which demands a relatively high concentration of mono- and divalent cations in solution to efficiently suppress electrostatic repulsion between backbone phosphate groups and to allow for folding into a 3D conformation that becomes competent for specific recognition and binding of a ligand. As a direct consequence, a pronounced dependence of oligonucleotide conformation and stability on the amount and type of counter-ions is found. It has been reported that alkali metal and alkali earth metal salts are often mandatory for aptamers to assure their functional properties ${ }^{55-56}$. Unfortunately, the preferred counter-ions, such as $\mathrm{Na}^{+}, \mathrm{K}^{+}, \mathrm{Ca}^{2+}$ and $\mathrm{Mg}^{2+}$, are poorly compatible with $\mathrm{ESI}$ due to salt-induced ion suppression and extensive clustering with the analyte ions. On the contrary, the effect of ESI-MS-compatible buffer salts, such as ammonium acetate, on the folding and binding 
properties of aptamers in solution is poorly characterized and is often considered detrimental. Nonetheless, several studies on the interaction of nucleic acids with $\mathrm{NH}_{4}^{+}$ ions suggested that the latter can effectively mimic $\mathrm{Na}^{+}$ions ${ }^{57-59}$. The formation of Gquadruplexes as a function of ammonium acetate concentration has been shown by native ESI-MS ${ }^{60-61}$. These data suggest that ammonium acetate may effectively stabilize aptamers in a folded conformation. Another related problem is a potentially high rate of nonspecific association between oligonucleotides and small polar or ionic ligands during ESI. Addressing these critical issues will allow broad application of native ESI-MS to study aptamers and will open new possibilities to systematic characterization of the properties of these exciting receptor molecules.

In this study, we demonstrate that nano-ESI-MS by itself or coupled with ion mobility spectroscopy (IMS), with properly adjusted experimental conditions, can be successfully used to determine the binding stoichiometry, specificity, selectivity, and affinity of aptamers. We correlate the native ESI-MS data, which is generally thought of as providing a "snapshot" of the solution-phase chemistry, to results of complementary circular dichroism (CD) and ITC measurements in solution under identical conditions to confirm the validity of the ESI-MS findings. We chose three well-studied aptamers with different structural features and mechanisms of ligand binding as model systems: an adenosine-binding aptamer (ABA), a L-argininamide-binding aptamer (LABA), and a cocaine-binding aptamer (CBA). All the major aspects of the model aptamer-ligand interactions are successfully captured in our native ESI-MS experiments, demonstrating the versatility and broad applicability of the approach.

\section{Experimental}

Materials and Methods. Aptamers specific for adenosine, L-argininamide, and cocaine as well as the respective negative-control oligonucleotides were purchased from 
Eurogentec (Geneva, Switzerland). The ligands employed in this study are depicted in Scheme 1. All chemicals used were of analytical grade or better and used without further purification. (see SI for the aptamer sequences and ligands).
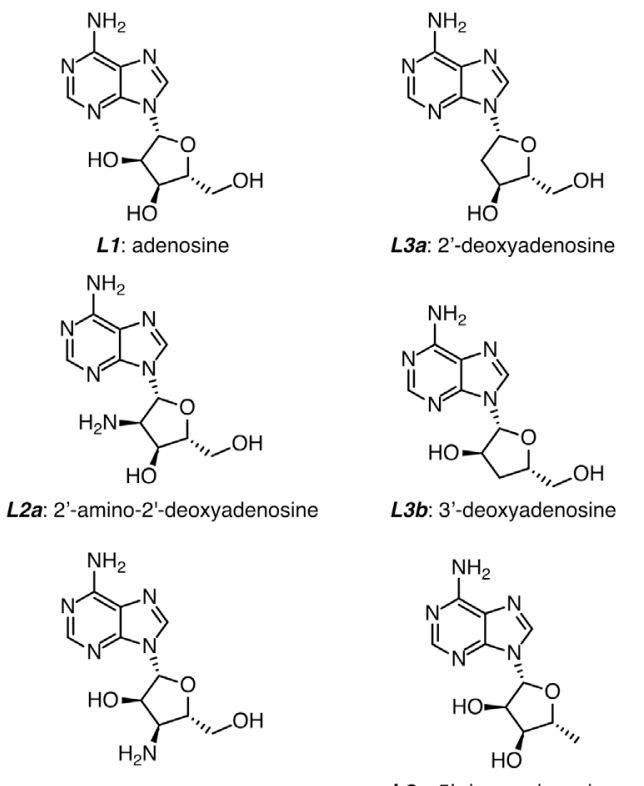

L2b: 3'-amino-3'-deoxyadenosine

L3c: 5'-deoxyadenosine
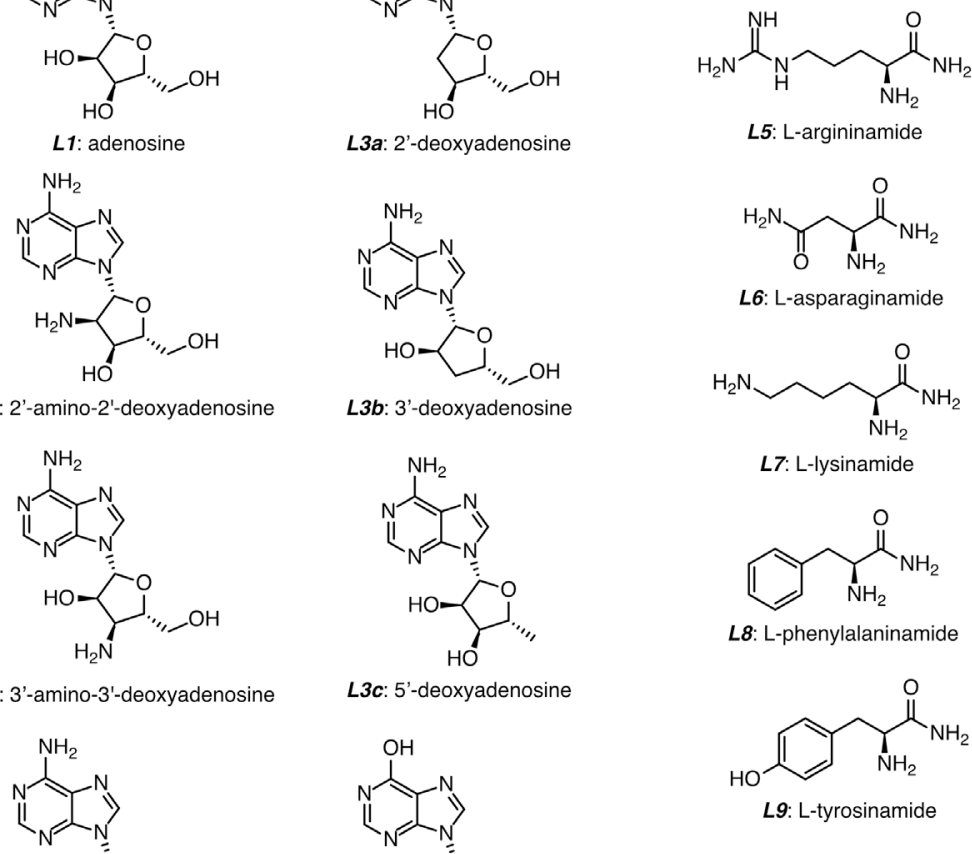

L5: L-argininamide

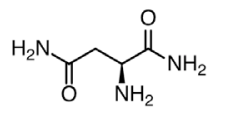

L6: L-asparaginamide

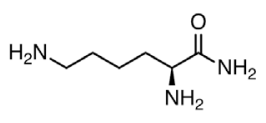

L7: L-lysinamide

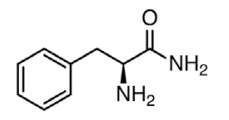

L8: L-phenylalaninamide

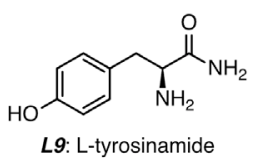

L2c: 5'-amino-5'-deoxyadenosine<smiles>OC[C@H]1O[C@@H](O)[C@@H](O)[C@@H]1O</smiles>

L4: inosine
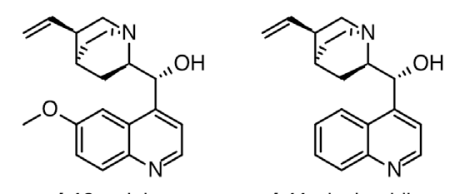

L11: cinchonidine

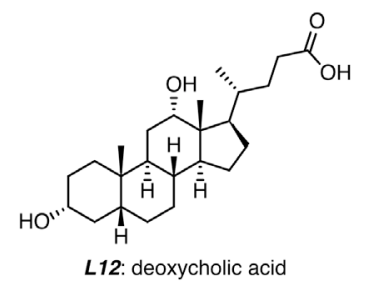

Scheme 1. Structures of the ligands studied.

\section{Mass Spectrometry.}

ESI mass spectra and ion mobility spectra were acquired with a hybrid quadrupole-timeof-flight mass spectrometer (Synapt G2-S HDMS, Waters, Manchester, UK) operated in the negative-ion mode. Ions were produced by a commercial nanoLock spray ionization source (Waters, Manchester, UK) using gold/palladium-coated borosilicate glass nanoESI emitters (Thermo Fisher Scientific, Reinach, Switzerland). Optimised ion source 
parameters were used to minimize in-source dissociation of the complexes (see SI text for details).

Isothermal Titration Calorimetry. Isothermal titration calorimetry experiments were performed on a Nano ITC calorimeter (TA Instruments, New Castle, USA; see SI text for method details).

Circular Dichroism Spectroscopy. Circular dichroism spectra were recorded on a Chirascan qCD spectropolarimeter (Applied Photophysics, UK) in the UV range of 200-320 $\mathrm{nm}$. (see SI text for method details)

\section{Results and Discussion}

Does nano-ESI-MS faithfully detect aptamer-ligand complexes and provide insight into the binding mechanism? Among the three different aptamers in this study, ABA and LABA have overall structures of a hairpin-loop and exhibit a high specificity towards their respective ligands, while CBA features three short stem regions with the ligand-binding region located at their junction. Interestingly, although CBA had been originally selected to specifically recognise and bind an illicit drug cocaine ${ }^{62}$, it was later found that the aptamer can bind a broad range of ligands ${ }^{63-64}$, some of which, e.g., quinine (L10, Scheme 1), with even greater affinity than cocaine ${ }^{65}$. High-resolution 3D structures of ABA and LABA determined by solution-phase NMR spectroscopy are available and reveal details of the aptamer-ligand recognition. ABA has two non-equivalent binding sites for adenosine $(L \mathbf{1}, \text { Scheme } 1)^{66}$. The NMR-derived structural model of LABA in complex with its ligand, L-argininamide ( $L 5$, Scheme 1$)$, suggests that the aptamer has a single ligand-binding pocket ${ }^{67}$. Both $A B A$ and LABA are thought to be rigid binders, i.e., to exist in solution in a stable binding-competent conformation that does not undergo any significant rearrangements upon ligand binding ${ }^{62-64}$. No high-resolution structural data is 
available for CBA, but the aptamer was reported to form a 1:1 complex with its ligands ${ }^{62}$. We used a truncated version of the originally selected CBA, which was reported to be loosely folded in solution due to a shortened stem region and undergo a ligand-induced folding-upon-binding transition ${ }^{68}$. By choosing these different aptamers, we expected to cover a spectrum of possible receptor-ligand interaction modes and thereby test the native ESI-MS approach in a variety of scenarios.

One very important variable to investigate was the influence of buffer solution composition on aptamer-ligand binding and the complex stability. We performed ESI-MS measurements of the aptamer solutions containing ammonium acetate at a concentration varied in the range of $50-500 \mathrm{mM}$ (Figure S1). At low ammonium acetate concentrations, all three aptamers were detected as a broad distribution of peaks corresponding to multiply charged ions. As the concentration of ammonium acetate increased, the charge state distributions (CSD) of aptamer ions gradually became narrower and shifted towards a lower average charge. While bivalent cations such as $\mathrm{Sr}^{2+}, \mathrm{Mg}^{2+}$ are sometimes tolerated in $\mathrm{ESI}$, the use of $\mathrm{Mg}^{2+}$ and $\mathrm{Mn}^{2+}$ as counter ions in our native nano-ESI experiments introduced significant adducts, which reduced spectral quality such that we decided to proceed with ammonium acetate alone. Complementary ITC and CD measurements were carried out under identical solution conditions.

Figure 1 presents ESI mass spectra and corresponding IMS mobilograms of ABA, $\angle A B A$, and CBA in the absence and presence of ligands, as well as non-binding analog sequences as control measurements. The ESI-IMS-MS measurements reveal a correlation between the charge state and conformation of electrosprayed aptamer ions (Figure 1a-c). For example, in the mobilogram of ABA (Figure 1a), the ion drift time increases rapidly and almost linearly with the decrease in the number of charges down to charge state 7-. At this point, the ion arrival time distribution (ATD) extends towards 
lower drift times, marking a transition from a more extended to a more compact conformation of the aptamer ions. As the number of charges decreases further, the conformation of ions converges to a compact folded state, which is reflected by the
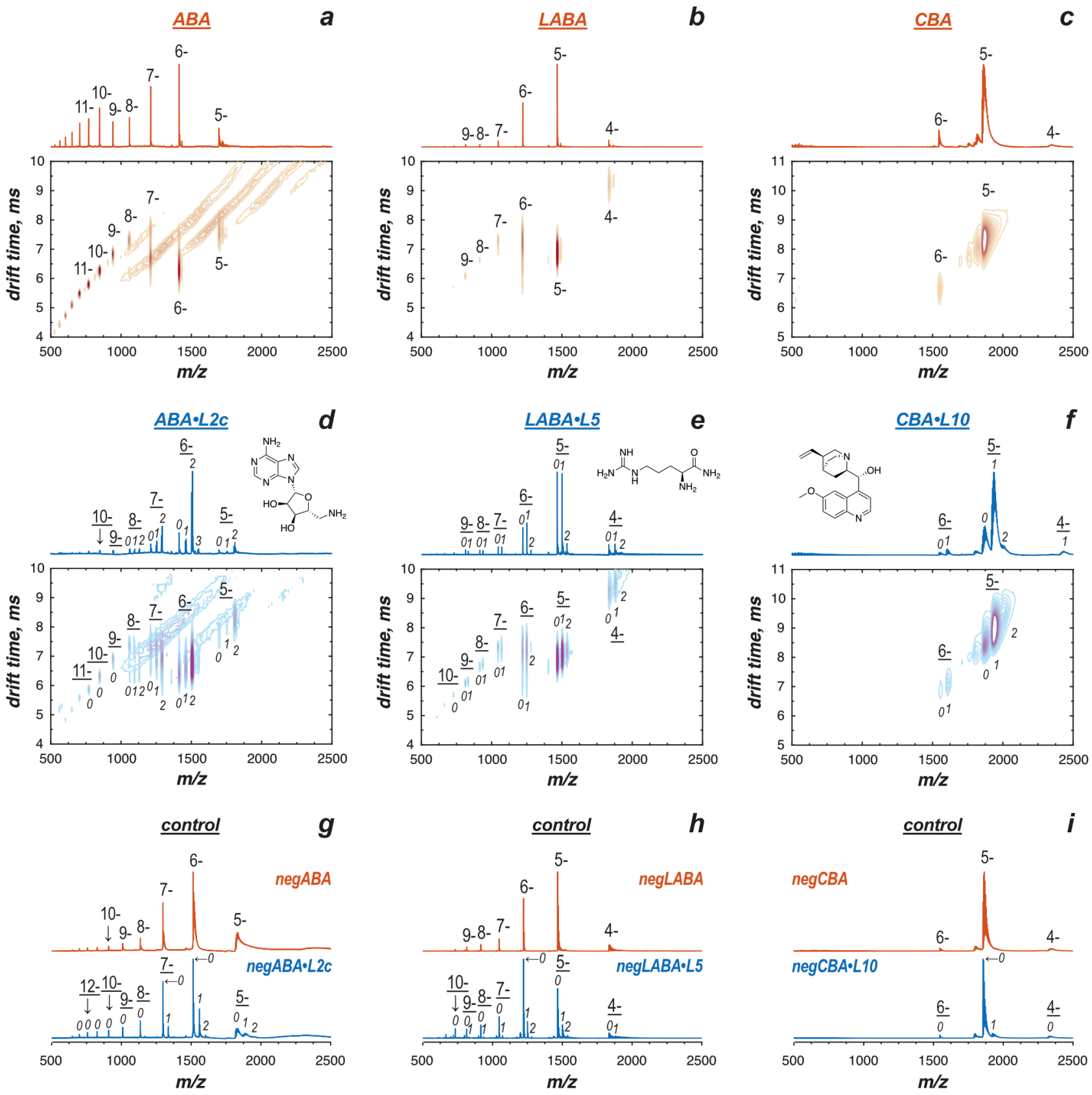

Figure 1. Native ion mobility-mass spectrometry (IM-MS) analysis of adenosine-binding (a), Largininamide-binding (b), and cocaine-binding (c) aptamers, as well as their complexes with specific ligands (d-f, respectively). Top traces in panels a-f represent mass spectra, and the contour plots below them show IM data (deeper colour corresponds to higher signal intensity). Charge states of ions are indicated above the respective peaks. The number of bound ligands is indicated in smaller italic numbers (d-i). Native ESI-MS spectra of non-binding analogs of the aptamers studied (controls) alone and mixed with the ligands are shown in panels $g$-i. Samples of adenosine-binding aptamer $(a, d)$ and L-argininamide binding aptamer (b, e), as well as of their non-binding analogues ( $\mathrm{g}, \mathrm{h}$, respectively) were prepared in 50 $\mathrm{mM}$ ammonium acetate buffer solution. Samples of cocaine-binding aptamer $(c, f)$ and its non-binding analogue (i) were made in $300 \mathrm{mM}$ ammonium acetate buffer solution. The aptamer concentration was 10 $\mu \mathrm{M}$ in all cases. 10 molar equivalents were mixed with adenosine-binding and L-argininamide-binding 
aptamer ( $d$, e, respectively) or to their non-binding analogues (bottom traces in $\mathrm{g}$ and $\mathrm{h}$, respectively). A five-fold molar excess of the ligand was used in $f$ and $i$.

observed narrowing of ATD. Similar trends were observed for LABA (Figure 1b) at low concentrations of ammonium acetate. In contrast, only a single pool of compact conformers existed when aptamers were electrosprayed from solutions containing a high concentration of ammonium acetate, as shown for example in Figure 1c for CBA. It is, however, unclear to what extent these observations reflect the structure of aptamers in solution. The processes of ionization, desolvation, and ion transfer in the gas phase may significantly affect the conformation of aptamers. In a recent study, Gabelica and coworkers demonstrated that DNA and RNA duplexes electrosprayed from solutions containing ammonium acetate at physiologically relevant concentrations $(\geq 100 \mathrm{mM})$, were significantly more compact in the gas phase compared to the canonical solution structures ${ }^{47}$. A similar compaction of DNA duplex during ESI was found in an extensive MD simulation study by Sharawy and Consta, provided that sufficient amount of $\mathrm{Na}^{+}$cations was present in an electrospray droplet whose net charge was less negative than $50 \%$ of the DNA charge ${ }^{69}$. Both studies concluded that isolated oligonucleotide ions retain some memory of their condensed-phase structure. Our own observations are consistent with these results. Clearly, further research combining both computational and experimental approaches is necessary to shed more light on the extent of preservation of oligonucleotide structure upon transfer from solution to the gas phase. Nevertheless, as we are demonstrating below, native ESI-MS can still provide an accurate snapshot of solution-phase aptamer-ligand interactions despite the significant influence of ESI on the aptamer conformational dynamics. 
We then incubated each aptamer with an excess of a ligand and measured them with native ESI-IMS-MS (Figure 1d-f). Compared to the spectra of bare aptamers, additional peaks emerged in the spectra, which were attributed to ions of aptamer-ligand complexes. For example, the spectrum of ABA mixed with 5'-amino-5'-deoxyadenosine (L2C in Scheme 1) featured a series of peaks corresponding to complexes with the aptamer-to-ligand ratios of 1:1, 1:2, and 1:3 (Figure 1d). Likewise, complexes of diverse stoichiometry were detected by ESI-MS for the other two aptamers: up to 1:3 for LABA, and up to 1:2 in the case of CBA (Figure 1e-f, respectively). Binding of more than one ligand has been unambiguously shown only for ABA, and LABA and CBA should form exclusively 1:1 complexes. Even though the most intense peaks in the spectra matched the complexes with the expected stoichiometry, the presence of complexes of aptamers with a higher than the expected number of ligands points to non-specific binding. To account for that, we measured native ESI-MS spectra of negative controls, i.e., oligonucleotides that are not expected to exhibit any specific binding of the ligands in solution under identical experimental conditions (Figure 19-i). We assumed that nonspecific association between oligonucleotides and small-molecule ligands is electrostatically driven and occurs to a similar extent for every oligonucleotide regardless of its sequence or structure ${ }^{70-71}$. As can be seen in Figure 19-i, complexes of the negative-control oligonucleotides with the ligands were detected with much lower signal intensity compared to the aptamer-ligand complexes in Figure 1d-f. We can therefore interpret the major signals in mass spectra as evidence for specific aptamer-ligand interactions in ammonium acetate-containing solutions, while only a minor fraction of the detected aptamer-ligand complexes arose due to non-specific binding during electrospray.

Remarkably, only low charge states were observed for the aptamer-ligand complex ions at low ammonium acetate concentrations (Figure 1d-e, S1c-e). For example, ABA・L2c 
complexes produced ions with the charge states ranging from 5- to 8-, while the bare aptamer ions carried up to 14 negative charges (Figure 1d). As discussed above, the lower charge states correspond to a more compact gas-phase conformation of the aptamers. Considering that folding of aptamers is required for ligand binding in solution, the preference of the detected complex ions for relatively low charge states further corroborates the specific nature of these complexes. Given that higher ammonium acetate concentrations facilitated aptamer folding in solution (see Figure S1a-b and discussion above), it seemed logical to assume a stabilizing effect of high salt on aptamer-ligand complexes. Surprisingly, we found an opposite trend for ABA and especially LABA (Figure S1c-d): the peak intensity of the complexes was significantly reduced or even vanished at higher ammonium acetate concentrations. Only in the case of CBA did we observe a rise in the complex peak intensity upon increase of ammonium acetate concentration (Figure S1e). Ammonium cations can form hydrogen bonds and ion pairs with DNA oligonucleotides, as is clearly reflected in ESI-MS data by peak broadening due to extensive ammonium cluster formation at high salt concentrations in ESI buffer solution. These interactions can outcompete the binding of polar and ionic ligands to aptamers, such as those of $A B A$ and $L A B A$. CBA in contrast recognizes relatively non-polar ligands. The contribution of hydrogen bonds and ion pairing in the binding of such ligands is likely less important. Hence, the CBA-ligand complex was almost insensitive to ammonium acetate (Figure S1e). Based on the presented evidence, we chose $50 \mathrm{mM}$ ammonium acetate concentration for the experiments on $A B A$ and $L A B A$, while the analyses of CBA were carried out at an ammonium acetate concentration of $300 \mathrm{mM}$, concentrations that agree with our previous findings ${ }^{72}$.

Another ESI artefact that might negatively affect our measurements and challenge our interpretation of the results is aptamer-ligand complex dissociation due to excessive 
collisional activation of ions in the ion source ${ }^{39}$, which would lead to complex dissociation predominantly in high charge states. Indeed, in tandem MS experiments on selectively isolated aptamer-ligand complex ions, the dissociation proceeded via a loss of neutral ligand molecules and occurred more readily in more highly charged ions (Figure S2). We therefore carefully optimized the ion source and collision cell parameters to minimize, if not eliminate, unwanted collision-induced dissociation of aptamer-ligand complexes during ion transfer through the mass spectrometer (Figure $\mathbf{S}$ ).

A very interesting finding for $A B A$, which we decided to explore further, was that not only did binding exclusively occur in lower charge states (4- to 7-), but also the intensity of more highly charged ions (7- to 12-) significantly decreased in the presence of ligand (Figure 1a, d). Furthermore, the shift in ATD towards shorter drift times was found already for the 8- ions and was more pronounced for the ligand-bound states (Figure S4).This suggests that the more compact, folded conformation of ABA is stabilized by the bound ligand under native ESI-IMSMS conditions. The presence of two distinct ABA conformations in solution has been revealed by single-molecule $\mathrm{FRET}^{73}$ and via real-time current measurement upon translocation of $\mathrm{ABA}$ through a hemolysin nanopore ${ }^{74}$. These studies unambiguously showed that ABA exists in two conformational states, where the folded form is populated even before any ligand is added, and that the dynamic equilibrium is shifted to the folded state upon an increase of salt and/or ligand concentration - a behaviour that is most consistent with the conformational selection mechanism. Our native ESI-IMS-MS results corroborate these findings. To study this further, we recorded complimentary circular dichroism (CD) spectra of the free and ligand-bound ABA in 50 $\mathrm{mM}$ and $300 \mathrm{mM}$ ammonium acetate buffer. As can be seen from Figure S5, the CD spectrum of free $A B A$ is only slightly different from that of $A B A$ in the presence of ligand and there is no direct evidence that a conformational rearrangement from an unfolded to a folded state is occurring. Thus, we believe that the addition of ligand did not induce any significant change in the overall structure of the aptamer in solution. Instead, a preformed folded structure is selected 
by the ligand. Unfortunately, the sensitivity of the CD measurements was not high enough to build a titration curve and demonstrate the shift of conformational equilibrium towards the folded state induced by the stabilizing effect of binding the ligand. Nonetheless, this effect was clearly observed by native ESI-IMS-MS (Figure 1a, d), which nicely highlights the advantageous high sensitivity of the method.

The preformed stable helical conformation capable of ligand binding was also postulated for $\mathrm{LABA}^{67}$. Unlike in ABA, the overall shape of CSD, as well as ion ATDs did not change much upon addition of the ligand (Figure 1b, e, Figure S4). Nevertheless, the preference of aptamerligand complexes for lower ion charge states was clearly observed in this case as well. LABA was originally found to form a hairpin loop, with residues important for binding positioned in the loop region ${ }^{75}$. Binding of $\mathbf{L} 5$ induces the formation of two non-Watson-Crick (G14-T10 and C5A7) and two Watson-Crick (C16-G9 and T17-A6) pairs, which facilitate generation of a binding pocket (Figure S10). Based on the structure of LABA predicted by $\mathrm{NMR}^{67}$, ligand binding does not change the folding behaviour of LABA completely, but still leads to a rearrangement of some of the base pair contacts. CD spectra also show that there is a pronounced intensity decrease of the peak at $280 \mathrm{~nm}$ after the addition of ligand, which reflects the changes in the base stacking before and after ligand binding (Figure S13). However, there was no distinct spectral change to indicate a drastic difference in the overall structure of the DNA aptamer by ligand binding. For an induced-fit binding, the energy of the interaction must be sufficient to overcome the energy barrier between the unfolded/nonbinding state to a folded/binding state, and consequently induce a conformational change. In a previous study on the energetic features of the LABA, it was found that this aptamer is selective but only binds weakly to the ligand ${ }^{76}$. It is therefore quite difficult to experimentally discriminate whether an aptamer adopts an induced fit binding mechanism, or a conformational selection mechanism operates during binding. A molecular dynamics study on the conformational dynamics of LABA showed that its 
conformation appears to undergo local changes in the binding pocket with distances of bases C9 to $\mathrm{C} 16$ and $\mathrm{G} 10$ to $\mathrm{C} 16$ increasing, and those between bases $\mathrm{A} 12$ and $\mathrm{C} 17$ decreasing during Arm binding. This MD study further found that the total binding energy from the formation of LABA-L5 complex was not the lowest value in whole process of DNA aptamer-ligand binding, which explains the binding ability of LABA even in the low energy regime. This was attributed to the entropic gain that may have arisen from induced-fit binding. ${ }^{77}$ Therefore, based on our data and these previous findings, it is clear that while a drastic conformational change was not evident, the ligand induces some changes in the binding pocket of LABA when binding.

Interestingly, the second ligand was quite strongly bound to LABA in ESI-MS (Figure 1b, e, Figure S2). While the reported solution-phase structure of the complex determined by NMR spectroscopy suggests a 1:1 stoichiometry, the same study also stated that the aptamer might contain more than one ligand-binding site because the argininamide lines in the NMR spectra were not consistent with a simple fast binding behaviour for a 1:1 complex ${ }^{67}$. Furthermore, in a recent molecular docking study on LABA, the relatively high number of clusters was reported to exist indicating the presence of multiple hotspots for the ligand binding ${ }^{78}$. This was attributed to the bis-cationic nature of $\mathbf{L} \boldsymbol{5}$ ligand. We therefore think that the $1: 2$ complex due to $\mathbf{L} \boldsymbol{5}$ binding might indicate a second weaker-affinity site in solution, rather than resulting from a non-specific adduct formation during ESI.

Figure 1c shows the results of native ESI-IMS-MS measurements for the cocaine-binding aptamer (CBA). The ESI mass spectrum of CBA consists mainly of three peaks corresponding to distinct ion charge states (4-, 5-, and 6- ). Upon incubation of the aptamer with a 5-fold molar excess of $L 10$ in 300 mM ammonium acetate, we clearly saw a strong signal for a 1:1 complex and a very low intensity peaks corresponding to a 1:2 complex (Figure 1f). The latter is attributed to a non-specific binding of the ligand to CBA in ESI, because a negative-control oligonucleotide, in which the proposed three-way junction structure of the CBA is disrupted, 
shows a peak for a non-specific 1:1 complex of nearly the same intensity as that of the 1:2 CBA $L 10$ signal (Figure 1i).

A ligand-induced folding-upon-binding mechanism was proposed for the CBA before ${ }^{62,79}$. It is quite noteworthy that although charge states from 5- to 9- are seen in the nano-ESI spectra acquired at $10 \mathrm{mM}$ and $50 \mathrm{mM}$ ammonium acetate, complex peaks appeared only at 6- and 5charge states. Moreover, there was no change in the charge state distribution upon adding increasing amounts of quinine to CBA (Figure S7). IM-MS maps show only negligible changes in ATD of 6- and 5- charge states of the CBA before and after ligand binding (Figure 1c, $\mathbf{f}$ and Figure S4). Previously reported ab initio reconstructions of free CBA and CBA $L 10$, as well as measurements of the radius of gyration $\left(R_{g}\right)$ also revealed only minor differences ${ }^{65}$. In another recent study, in contrast to the predominant view of the binding mechanism of CBA, this aptamer was reported to undergo minor structural changes upon ligand binding ${ }^{80}$. A new report $^{81}$ on the stem length and stability in several CBA variants showed by NMR spectroscopy of imino protons that the sequence we used in this study (referred to as MN1968) has a partially preformed stem. The NMR data was insufficient to resolve the ensemble of unstructured/structured states of CBA due to the fast hydrogen exchange with bulk water in the very dynamic stem region. Nonetheless, the ligand binding occurred more readily in the case of dramatically better-structured, ordered aptamer.

When we carried out CD measurements in solution at two different ammonium acetate concentrations, the intensity of the peak at $280 \mathrm{~nm}$ decreased after the addition of ligand. (Figure S16). However, the spectral features of CBA before and after ligand binding were nearly the same, i.e., the aptamer does not undergo a large change in secondary structure. Taken together, the previous results and the present nano-ESI-MS, IMS, and CD data suggest that the ligand more likely binds to a pre-ordered CBA conformer rather than induces a dramatic structural change in the aptamer. 
What do nano-ESI data tell about ligand selectivity of the studied aptamers? As the next step, we wanted to see if the selectivity of aptamers can be probed by nano-ESI-MS. In the case of ABA, we used eight different structural analogues of adenosine shown in Scheme 1. We incubated $5 \mu \mathrm{M} \mathrm{ABA}$ and a 5 -fold molar excess of the respective ligands and performed nano-ESI measurements. The data presented in Figures 2a and S6a clearly shows that the affinity order was $L 2 c>L 2 b>L 1>L 2 a>L 3 b>L 3 c \approx L 3 a>L 4$. Surprisingly, there was almost no binding for all deoxyadenosine analogues, and only a very small signal of the 1:1 complex. This suggests that the hydroxyl groups of the sugar ring play an essential role in the binding, and irrespective of the position in the ring, binding is lost upon dehydroxylation. The importance of $\mathrm{H} 1\left(1^{\prime}-\mathrm{OH}\right)$ and $\mathrm{H} 4\left(4^{\prime}-\mathrm{OH}\right)$ protons for correct ligand orientation in the binding pocket has been revealed by NMR spectroscopy (Figure S8) ${ }^{66}$. Our observations agree well with these results.
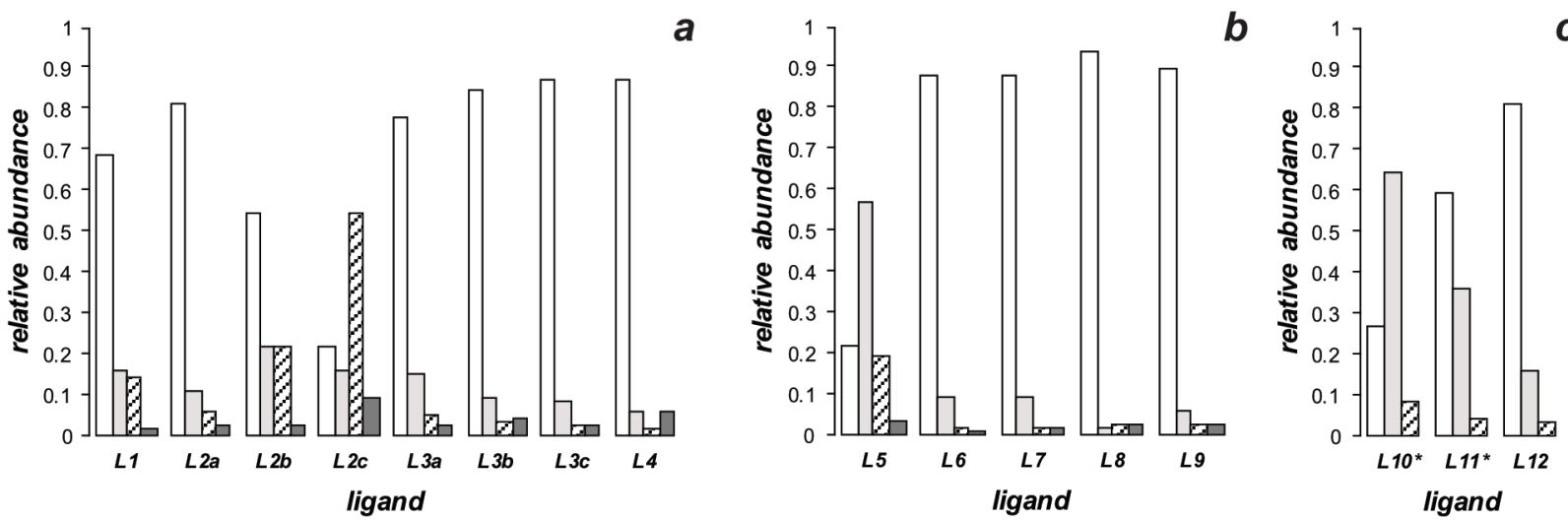

$\square A L_{0} \quad \square A L_{1} \quad \square A L_{2} \quad \square A L_{3}$

Figure 2. Native nano ESI-MS analysis of ABA (a), LABA (b), and CBA (c), in the presence of various ligands (L1L12, respectively; see Scheme 1 for chemical structures of the ligands). * $L 10$ and $L 11$ were added to CBA at 5fold molar excess. In all other cases, 10 -fold molar excess of the ligand was used.

To study the ligand selectivity of LABA, we selected five different amino acid amides ( $\mathbf{5}$ - L9, Scheme 1). As seen in Figures $\mathbf{2 b}$ and $\mathbf{S 5 b}$, the aptamer efficiently discriminated against nontarget amino acid amides, with only minor peaks indicating complex formation with lysinamide (L7). The major structural difference between $\mathbf{L 7}$ and $\mathbf{L} \mathbf{5}$ is that the latter contains a guanidine 
group. LABA forms a hairpin loop, with residues important for binding positioned in the loop region. Binding of $\boldsymbol{L} 5$ induces the formation of two non-Watson-Crick (G14-T10 and C5-A7) and two Watson-Crick pairs (C16-G9 and T17-A6), which facilitate generation of a binding pocket. The specificity for $\boldsymbol{L} \boldsymbol{5}$ seems to arise from contacts between the guanidine moiety of the ligand and phosphates of the aptamer backbone, with their atoms appropriately positioned by the shape of the pocket.

As noted previously, CBA has a broad ligand selectivity and binds better to the ligands for which it was not originally selected. Such a broad ligand specificity is quite uncommon for aptamers and is a remarkable feature of CBA. We incubated quinine, cinchonidine, and deoxycholic acid (L10, L11, and L12 in Scheme 1, respectively) with CBA. In Figure 2c, we see that the binding affinity of CBA towards $L 11$ was greatly reduced. The only structural difference between $L 11$ and $L 10$ is the lack of a methoxy group on the aromatic ring (Scheme 1). In a previous ITCbased study, the binding affinity of CBA was found to be reduced four-fold from $L 10$ to $L 11^{82}$. The bile acid $\mathbf{L} \mathbf{1 2}$, although being structurally very different from the other two ligands, was also found as a ligand that CBA recognizes, but with a lower affinity ${ }^{83}$. However, as seen in Figures 2c and S5c, the amount of detected CBA $\cdot$ L12 complex was strongly diminished. According to the binding mechanism proposed for $\mathrm{CBA}^{62,84}$, bending of the $\mathrm{T} 21$ over $\mathrm{C} 20$ creates a binding pocket. Therefore, in the light of previous reports and of our data we think that CBA shows an affinity towards the ligands that could be encaged into this pocket.

Determination of aptamer-ligand binding constants with native ESI-MS and ITC. As unambiguously shown above, aptamer-ligand complexes can be successfully probed, and specific and nonspecific interactions can be discriminated by nano-ESI-MS. Furthermore, the peak intensity in mass spectra correlate with the abundances of respective species in the reaction mixture and reflect the strength of binding. Below we use this information to quantify the binding affinity of aptamer-ligand complexes. The underlying assumption is that ionization 
efficiencies of the bare receptor and receptor-ligand complexes are identical, which is generally the case when the size of the ligand is small compared to the receptor. We also incorporated control sequences for all three aptamers to take into account any changes that might arise from non-uniform response factors. We used this data in the mathematical calculations.

As discussed above, there are two distinct ligand-binding sites in ABA. The aptamer-ligand binding equilibrium of $A B A$ in solution can formally be described as a two-step reversible reaction,

$$
\begin{aligned}
& L \quad L \\
& A \rightleftarrows A L \rightleftarrows A L_{2} \\
& \downarrow \uparrow L \quad \downarrow \uparrow L \quad \downarrow \uparrow L \\
& A l \quad A L l \quad A L_{2} l \quad \text { (Equation 1) }
\end{aligned}
$$

where $L$ is the ligand and $A, A L$, and $A L_{2}$ are the free aptamer and specific complexes of the aptamer with one and two specifically bound ligands, respectively. Due to up-concentration of analytes in electrospray droplets, the ligand molecules can also bind non-specifically to the aptamers, which manifests itself most clearly by the emergence of peaks corresponding to a complex of the aptamer with a third ligand bound in mass spectra recorded at higher ligand concentrations. This is symbolized by a small letter $\ell$ in the equations. We assume that such non-specific binding occurs equally for the free aptamer molecules and those that have already specifically bound one or two ligands. Since no aptamer-ligand complexes with more than three bound ligands were detected, and because only 1:1 binding was detected in the negativecontrol data, we conclude that at most one non-specific binding event was occurring on the time scale of the experiment, giving rise to complexes $A \ell, A L \ell$, and $A L_{2} \ell$ (Equation 1). We performed the titration experiments using $L 2 c$, which was shown to bind to ABA most strongly (Figure 2), and obtained the titration curves shown in Figure 3. We then used the mathematical equations derived in the SI (equations 2-9). 
To obtain the binding constants, we considered each charge state separately or alternatively combined peak intensities of all charge states. The data is represented in Figures $\mathbf{3}$ and $\mathbf{S 9}$, as well as in Tables $\mathbf{1}$ and S4. Using only the charge states 5 - and 6- resulted in a better fit. This is because of the higher signal intensity and the better signal-to-noise ratio found for the lower charge states. Furthermore, as discussed above, aptamer-ligand interactions were better preserved in the complex ions of lower charge states while the complex ions of higher charge states were more prone to dissociation in the gas phase. We calculated the $K_{d}$ values for 1:1 and 1:2 ABA.L2c complexes to be of $69.7 \pm 7.9 \mu \mathrm{M}$ and $5.3 \pm 0.6 \mu \mathrm{M}$, respectively (Table 1). It is already clear from the nano-ESI spectrum shown in Figure 1 that the 1:2 complex has a much higher intensity than the 1:1 complex, i.e., a positive cooperativity exists. Indeed, the highresolution structure of $A B A$ complex with the ligand was previously resolved by solution-phase NMR spectroscopy and it suggested that this aptamer has two non-equivalent binding sites ${ }^{66}$.

We also tested other binding models, particularly, to assess whether or not a distinction between the induced fit and conformation selection mechanisms could be made based on the ESI-MS titration data alone (Schemes S1-S3 and Tables S2-S3). However, without an independent measurement of the fraction of the unfolded aptamer, the unfolding constant $K_{f}$ cannot be determined with high confidence simultaneously with the binding constants, due to the interdependence of the parameters. The most direct approach to discriminate between induced fit and conformational selection is the determination of the kinetic rate constants of each individual binding reaction ${ }^{85-87}$, but ESI-MS titration measurements reported here were performed at equilibrium conditions and do not give access to kinetic rate constants. Hence, we did not consider the aptamer folding equilibrium further in the binding models applied to LABA and CBA.

A $K_{d}$ of $6 \pm 3 \mu \mathrm{M}$ for adenosine had previously been determined by analytical ultracentrifugation experiments $^{88}$. In another recent example, optical tweezers and equilibrium capillary 
electrophoresis (CE) were used, and $K_{d}$ values of $2.0 \pm 0.2 \mu \mathrm{M}$ and $2.4 \pm 0.4 \mu \mathrm{M}$, respectively, were found ${ }^{89}$. However, these studies were conducted in the original SELEX buffer solution, which is different from the native ESI-MS buffer solution. To learn how aptamer-ligand binding was affected by the use of ammonium acetate as a buffer salt, and if the nano-ESI-MS measurements were in agreement with the solution-phase measurements, we performed isothermal titration calorimetry measurements at conditions identical to those of the nano-ESIMS, which is, using $50 \mathrm{mM}$ ammonium acetate as a buffer. While it is possible that aptamerligand binding is different in a buffer that lacks $\mathrm{Na}^{+}, \mathrm{K}^{+}$and $\mathrm{Mg}^{2+}$ ions, it was very clear from the ITC results (Figure 3) that the solution-phase $K_{d}=8.0 \mu \mathrm{M}$ was in a good agreement with the previous solution-phase data. Noteworthy, the data from our ITC measurements, as well as previous solution-phase methods, gave only a single $K_{d}$ value. A recent systematic ITC study on different structural variants of $A B A$ was able to resolve 2 binding sites, but only reported a single value of $K_{d}=16.4 \pm 1.4 \mu \mathrm{M}^{90}$. Our attempts to apply a different binding model to the ITC data to account for multiple ligand binding and cooperativity failed to produce a high-quality fit. A more extensive ITC study could potentially resolve this issue but would require a much greater sample amount. In contrast, the native ESI-MS titration readily affords a more appropriate and accurate binding model, which is consistent with the high-resolution 3D structure of the aptamer-ligand complex.

Since the existence of second binding site for LABA is controversial, we assumed one strong and one weak solution-phase binding step for the LABA producing the following species in solution (Equation 2, bold font): the bare aptamer $(\boldsymbol{A})$, the strongly bound specific complex $(\boldsymbol{A L})$, the weak solution-phase complex $(\ell \boldsymbol{A})$, and the complex with both the strong and weak binding sites occupied by the ligand (eAL). Each of these species, according to our analysis (see above), could bind up to two additional ligands nonspecifically during ESI (designated as 
small " $l$ " added to the end of expressions for the solution-phase species listed above; Equation 2).

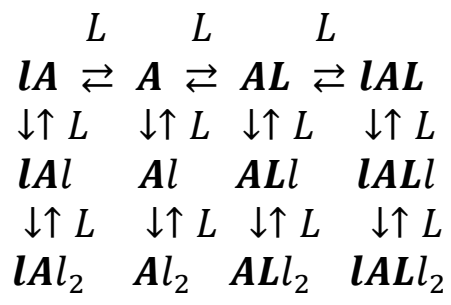

\section{(Equation 2)}

Associated data and the titration curves are depicted in Table 1, Table S5, and in Figure 3, Figure S11-S12. We found 22.04 $\mu \mathrm{M}$ and $179.0 \mu \mathrm{M}$ for the dissociation constants for 1:1 and "pseudo" 1:2 LABA•L5 complexes. To compare the nano-ESI derived $K_{d}$ results with solutionphase measurements, ITC titration was performed under the same buffer conditions as in nanoESI (Figure 3). The ITC derived $K_{d}$ was found to be $293.1 \mu \mathrm{M}$, which was close to the previously reported values of $166 \mu \mathrm{M}$ and $164 \mu \mathrm{M}$ obtained by CD and DSC measurements ${ }^{76}$. In the case of LABA, the $K_{d}$ values derived from the nano-ESI data differed slightly from both the ITC data and previous literature findings. Noteworthy, both the second "pseudo" binding and non-specific binding were quite strong, with $K_{d}$ values of $179.0 \mu \mathrm{M}$ and $274.0 \mu \mathrm{M}$, respectively, which we attribute to the strengthening of ion-pair interactions between the cationic ligand and the aptamer upon desolvation and transfer to the gas phase.

$$
\begin{array}{lll} 
& \multicolumn{2}{c}{L} \\
\boldsymbol{A} & \boldsymbol{A L} & \\
\downarrow \uparrow & \quad \downarrow \uparrow L & \\
\boldsymbol{A l} \quad \boldsymbol{A L l} & \text { (Equation 3) }
\end{array}
$$

Finally, we calculated the dissociation constants of CBA $\angle 10$ by assuming one specific solutionphase binding step and one nonspecific binding step during ESI-MS (Equation 3). The results are summarized in Table 1 and Table S6. As derived from the titration curves (Figure $\mathbf{3}$ and Figure S15), the $K_{d}$ of the CBA-L10 interaction was found to be $8.5 \mu \mathrm{M}$. The binding for the second non-specific binding was much weaker, which further suggests that the existence of second binding site is unlikely. To compare the nano-ESI derived $K_{d}$ with a solution-phase 
reference value, we performed ITC at identical conditions. Figure 3 shows the ITC curve of the CBA. A $K_{d}$ of $2.3 \mu \mathrm{M}$ was found, in good agreement with the nano-ESI data. The previous ITCderived $K_{d} \approx 0.23 \mu \mathrm{M}$ obtained in the original SELEX buffer ${ }^{65}$ is lower than the values obtained here, most likely due to the different buffer conditions used.

Table 1. Dissociation constants determined by nonlinear least-square fitting of the direct nano ESI-MS titration data

\begin{tabular}{|c|c|c|c|c|c|c|c|}
\hline \multirow{3}{*}{ Aptamer } & \multicolumn{6}{|c|}{ This work } & \multirow{3}{*}{$\begin{array}{l}\text { Literature } \\
\qquad K_{d}(\mu \mathrm{M})\end{array}$} \\
\hline & \multicolumn{5}{|c|}{ Native ESI-MS titration } & \multirow{2}{*}{$\begin{array}{c}\text { ITC } \\
K_{d}(\mu \mathrm{M})\end{array}$} & \\
\hline & Charge state(s) & $K_{d 1}(\mu \mathrm{M})$ & $K_{d 2}(\mu \mathrm{M})$ & $K_{n}(\mu \mathrm{M})^{\mathrm{a}}$ & $S S R^{b}$ & & \\
\hline $\mathrm{ABA}$ & 5- to $6-$ & $69.7 \pm 7.9$ & $5.3 \pm 0.6$ & $841.0 \pm 136.8$ & 0.0325 & $8.0 \pm 1.2$ & $2.0 \pm 0.2^{76,89}$ \\
\hline LABA & 4- to $7-$ & $22.2 \pm 0.9$ & $179.0 \pm 25.8$ & $274.0 \pm 34.5$ & 0.0199 & $293.1 \pm 280.3$ & $166.7 \pm 10.8^{76}$ \\
\hline CBA & 4- to 6- & $8.5 \pm 1.5$ & NA & $104.6 \pm 37.3$ & 0.0875 & $2.3 \pm 1.0$ & $0.23 \pm 0.03^{65}$ \\
\hline
\end{tabular}

${ }^{\mathrm{a}} K_{n}$ - dissociation constant for the non-specific binding step during ESI (see Equations 1-3)

${ }^{b}$ SSR - sum of squared residuals (smaller is better) 

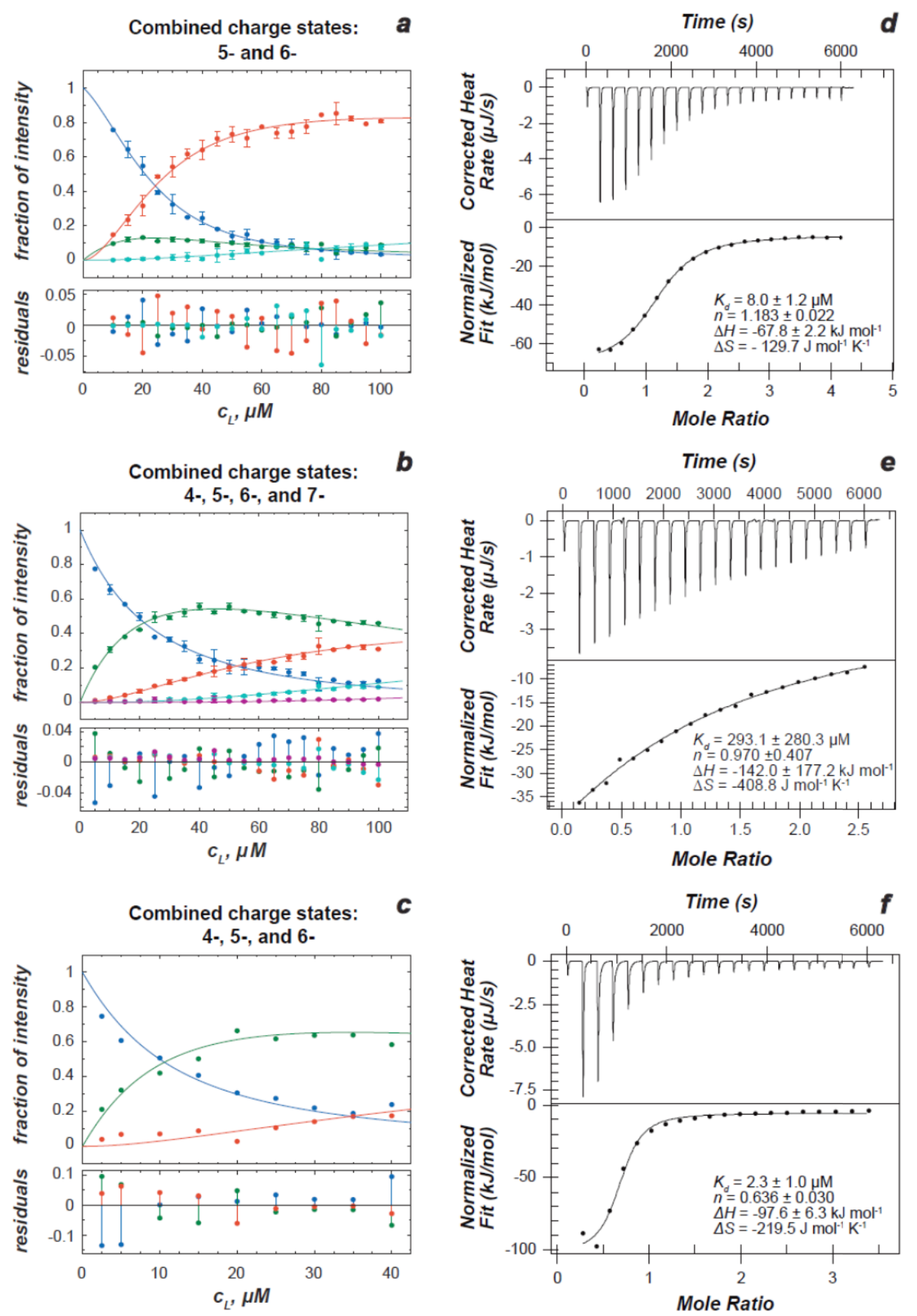

Figure 3. Nonlinear least-square fitting of the direct ESI-MS-based titration data and associated ITC curves obtained at identical solution conditions for ABA (a, d), LABA (b, e) and CBA (c, f). In the ESI-MS experiments, the aptamer concentration was kept at $5 \mu \mathrm{M}$. Various amounts of the respective ligands were incubated with the aptamer, and the mixtures were then analysed by nano-ESI-MS. The peak integrals were measured from the mass spectra and used to calculate the relative abundance of the free $\left(A L_{0}\right.$, blue) and ligand-bound $\left(A L_{1}, A L_{2}, A L_{3}\right.$, and $A L_{4}$ shown in green, orange, cyan, and purple, respectively) aptamer at equilibrium in solution. Individual data points are shown as dots with error bars, where applicable. In ITC, twenty 2.45- $\mu \mathrm{l}$ aliquots of 1-mM ligand solution were mixed with a fixed concentration of aptamer in the measurement cell: $79 \mu \mathrm{M}$ ABA, $129 \mu \mathrm{M}$ LABA, and $100 \mu \mathrm{M}$ CBA. An independent ligand binding model was fitted to baseline-corrected data to determine parameter values (shown with 95-\% confidence intervals). 


\section{Conclusions}

We performed a systematic study of three different DNA-aptamers by nano-ESI-MS, IMS, ITC, and CD. We show that with careful experimental design, native ESI-MS can deliver a wealth of fundamentally important information about aptamer-ligand interactions. This includes the stoichiometry of aptamer-ligand complexes, specificity of binding, ligand selectivity, and cooperative effects in aptamer-ligand binding. Our results, together with some previously reported evidence, strongly indicate that ligand binding in the aptamers studied herein most likely proceeds via conformational selection and not according to the widely accepted induced fit mechanism. The question of whether induced fit or conformational selection is generally operative in receptor-ligand interactions is a subject of hot debate. Unambiguous discrimination of the binding mechanism is only possible with relaxation kinetics experiments and by relating the $k_{\text {on }}$ and $k_{\text {off }}$ to the apparent binding constants ${ }^{85,91-94}$, which is currently a challenge for the native ESI-IMS-MS. Another limitation of the native MS approach is incomplete understanding of the effects that ESI has on the conformation and dynamics of aptamers. Nevertheless, native ESI-MS still provides an accurate snapshot of the solution-phase binding equilibrium even if the structure of an aptamer-ligand complex is affected by ESI, as long as experimental conditions are optimised to allow the complexes to remain bound long enough to be detected.

ESI-MS allows for refining the model for aptamer-ligand binding equilibrium, thanks to direct access to the information on the complex stoichiometry and cooperativity effects. This renders ESI-MS superior to ITC and other solution-phase methods, which fail to capture the full complexity of binding equilibrium. ${ }^{95}$ Nano-ESI-MS provides access to direct label-free quantitation of aptamer affinity to ligands, it also allows for an easy ranking of ligands and/or aptamers by their affinity. Considering that SELEX usually yields multiple aptamer candidates, ESI-MS could become a highly complementary approach for screening aptamers and selecting the top hit. 
Our study is certainly not exhaustive. We limited the choice of model systems to three DNAaptamers that were previously extensively studied by earlier established solution-phase biophysical techniques. However, the repertoire of aptamers includes RNA-aptamers that can also incorporate modified and non-standard nucleobases expanding and improving binding characteristics, and even peptide aptamers. Such a broad variety of aptamers is expected to exhibit highly diverse types of behaviour, which may differ significantly from those described here. Nonetheless, the approach described here can be applied to those systems as well and, as we showed in the cases studied here, may deliver important insights into the mechanisms of aptamer-ligand interactions.

\section{Acknowledgments}

We thank the Swiss National Science Foundation (grant no. 200020_159929) for financial support. BG thanks the ETH Zürich and the EU Marie Curie Co-Fund programme for a postdoctoral fellowship. 


\section{References}

1. Tuerk, C.; Gold, L., Systematic evolution of ligands by exponential enrichment: RNA ligands to bacteriophage T4 DNA polymerase. Science 1990, 249 (4968), 505.

2. Ellington, A. D.; Szostak, J. W., In vitro selection of RNA molecules that bind specific ligands. Nature 1990, 346 (6287), 818-822.

3. Dunn, M. R.; Jimenez, R. M.; Chaput, J. C., Analysis of aptamer discovery and technology. Nature Reviews Chemistry 2017, 1, 0076.

4. Zhou, J.; Rossi, J., Aptamers as targeted therapeutics: current potential and challenges. Nature Reviews Drug Discovery 2016, 16, 181.

5. Bjerregaard, N.; Andreasen, P. A.; Dupont, D. M., Expected and unexpected features of protein-binding RNA aptamers. Wiley Interdisciplinary Reviews: RNA 2016, 7 (6), 744-757.

6. Gelinas, A. D.; Davies, D. R.; Janjic, N., Embracing proteins: structural themes in aptamer-protein complexes. Current Opinion in Structural Biology 2016, 36, 122-132.

7. Ruigrok, V. J. B.; Levisson, M.; Hekelaar, J.; Smidt, H.; Dijkstra, B. W.; van der Oost, J., Characterization of Aptamer-Protein Complexes by X-ray Crystallography and Alternative Approaches. International Journal of Molecular Sciences 2012, 13 (8), 10537.

8. Tesmer, J. J. G., Crystallographic Pursuit of a Protein-RNA Aptamer Complex. In Nucleic Acid Aptamers: Selection, Characterization, and Application, Mayer, G., Ed. Springer New York: New York, NY, 2016; pp 151-160.

9. Qin, F.; Ye, W.; Chen, Y.; Chen, X.; Li, Y.; Zhang, J.; Chen, H.-F., Specific recognition between intrinsically disordered LEF and DNA. Physical Chemistry Chemical Physics 2012, 14 (2), 538-545.

10.Cragnolini, T.; Derreumaux, P.; Pasquali, S., Coarse-Grained Simulations of RNA and DNA Duplexes. The Journal of Physical Chemistry B 2013, 117 (27), 8047-8060.

11.Perez, A.; Orozco, M., Real-Time Atomistic Description of DNA Unfolding. Angewandte Chemie International Edition 2010, 49 (28), 4805-4808.

12.Dršata, T.; Zgarbová, M.; Jurečka, P.; Šponer, J.; Lankaš, F., On the Use of Molecular Dynamics Simulations for Probing Allostery through DNA. Biophysical Journal 110 (4), 874-876.

13.van der Vaart, A., Coupled binding-bending-folding: The complex conformational dynamics of protein-DNA binding studied by atomistic molecular dynamics simulations. Biochimica et Biophysica Acta (BBA) - General Subjects 2015, 1850 (5), 1091-1098.

14.Kolář, M.; Kubař, T.; Hobza, P., Sequence-Dependent Configurational Entropy Change of DNA upon Intercalation. The Journal of Physical Chemistry B 2010, 114 (42), 13446-13454.

15.Portella, G.; Orozco, M., Multiple Routes to Characterize the Folding of a Small DNA Hairpin. Angewandte Chemie International Edition 2010, 49 (42), 7673-7676.

16.Gkionis, K.; Kruse, H.; Platts, J. A.; Mládek, A.; Koča, J.; Šponer, J., Ion Binding to Quadruplex DNA Stems. Comparison of MM and QM Descriptions Reveals Sizable Polarization Effects Not Included in Contemporary Simulations. Journal of Chemical Theory and Computation 2014, 10 (3), 1326-1340.

17.Jayapal, P.; Mayer, G.; Heckel, A.; Wennmohs, F., Structure-activity relationships of a caged thrombin binding DNA aptamer: Insight gained from molecular dynamics simulation studies. Journal of Structural Biology 2009, 166 (3), 241-250.

18.Torabi, R.; Bagherzadeh, K.; Ghourchian, H.; Amanlou, M., An investigation on the interaction modes of a single-strand DNA aptamer and RBP4 protein: a molecular dynamic simulations approach. Organic \& Biomolecular Chemistry 2016, 14 (34), 8141-8153.

19.Xiao, J.; Salsbury, F. R., Molecular dynamics simulations of aptamer-binding reveal generalized allostery in thrombin. Journal of Biomolecular Structure and Dynamics 2016, 1-16.

20.Zeng, X.; Zhang, L.; Xiao, X.; Jiang, Y.; Guo, Y.; Yu, X.; Pu, X.; Li, M., Unfolding mechanism of thrombin-binding aptamer revealed by molecular dynamics simulation and Markov State Model. 2016, 6, 24065.

21.Villa, A.; Wöhnert, J.; Stock, G., Molecular dynamics simulation study of the binding of purine bases to the aptamer domain of the guanine sensing riboswitch. Nucleic Acids Research 2009, 37 (14), 4774-4786.

22. Hilder, T. A.; Hodgkiss, J. M., The Bound Structures of 17ß-Estradiol-Binding Aptamers. ChemPhysChem 2017, 18 (14), 1881-1887. 
23.Sun, L.; Jin, H.; Zhao, X.; Liu, Z.; Guan, Y.; Yang, Z.; Zhang, L.; Zhang, L., Unfolding and Conformational Variations of Thrombin-Binding DNA Aptamers: Synthesis, Circular Dichroism and Molecular Dynamics Simulations. ChemMedChem 2014, 9 (5), 993-1001.

24.Gong, Z.; Zhao, Y.; Chen, C.; Duan, Y.; Xiao, Y., Insights into Ligand Binding to PreQ1 Riboswitch Aptamer from Molecular Dynamics Simulations. PLOS ONE 2014, 9 (3), e92247.

25.Ditzler, M. A.; Otyepka, M.; Šponer, J.; Walter, N. G., Molecular Dynamics and Quantum Mechanics of RNA: Conformational and Chemical Change We Can Believe In. Accounts of Chemical Research 2010, 43 (1), 40-47.

26.Zhang, D.; Lu, M.; Wang, H., Fluorescence Anisotropy Analysis for Mapping Aptamer-Protein Interaction at the Single Nucleotide Level. Journal of the American Chemical Society 2011, 133 (24), 9188-9191.

27.Li, Y.; Sun, L.; Zhao, Q., Competitive fluorescence anisotropy/polarization assay for ATP using aptamer as affinity ligand and dye-labeled ATP as fluorescence tracer. Talanta 2017, 174, 7-13.

28.Spiga, F. M.; Maietta, P.; Guiducci, C., More DNA-Aptamers for Small Drugs: A Capture-SELEX Coupled with Surface Plasmon Resonance and High-Throughput Sequencing. ACS Combinatorial Science 2015, 17 (5), 326-333.

29.Sun, W.; Song, W.; Guo, X.; Wang, Z., Ultrasensitive detection of nucleic acids and proteins using quartz crystal microbalance and surface plasmon resonance sensors based on target-triggering multiple signal amplification strategy. Analytica Chimica Acta 2017, 978, 42-47.

30.Minagawa, H.; Onodera, K.; Fujita, H.; Sakamoto, T.; Akitomi, J.; Kaneko, N.; Shiratori, I.; Kuwahara, M.; Horii, K.; Waga, I., Selection, Characterization and Application of Artificial DNA Aptamer Containing Appended Bases with Sub-nanomolar Affinity for a Salivary Biomarker. 2017, 7, 42716.

31.Vogel, M.; Suess, B., Label-Free Determination of the Dissociation Constant of Small Molecule-Aptamer Interaction by Isothermal Titration Calorimetry. In Nucleic Acid Aptamers: Selection, Characterization, and Application, Mayer, G., Ed. Springer New York: New York, NY, 2016; pp 113-125.

32.Chen, B.; LeBlanc, R.; Dayie, T. K., SAM-II Riboswitch Samples at least Two Conformations in Solution in the Absence of Ligand: Implications for Recognition. Angewandte Chemie International Edition 2016, 55 (8), $2724-$ 2727.

33.Zhao, B.; Zhang, Q., Characterizing excited conformational states of RNA by NMR spectroscopy. Current Opinion in Structural Biology 2015, 30 (Supplement C), 134-146.

34.Yee, A. A.; Savchenko, A.; Ignachenko, A.; Lukin, J.; Xu, X.; Skarina, T.; Evdokimova, E.; Liu, C. S.; Semesi, A.; Guido, V.; Edwards, A. M.; Arrowsmith, C. H., NMR and X-ray Crystallography, Complementary Tools in Structural Proteomics of Small Proteins. Journal of the American Chemical Society 2005, 127 (47), 16512-16517.

35.Gülbakan, B.; Barylyuk, K.; Zenobi, R., Determination of thermodynamic and kinetic properties of biomolecules by mass spectrometry. Current Opinion in Biotechnology 2015, 31, 65-72.

36.Lössl, P.; van de Waterbeemd, M.; Heck, A. J. R., The diverse and expanding role of mass spectrometry in structural and molecular biology. The EMBO Journal 2016, 35 (24), 2634.

37.Leney, A. C.; Heck, A. J. R., Native Mass Spectrometry: What is in the Name? Journal of The American Society for Mass Spectrometry 2017, 28 (1), 5-13.

38.Gabelica, V.; Galic, N.; Rosu, F.; Houssier, C.; De Pauw, E., Influence of response factors on determining equilibrium association constants of non-covalent complexes by electrospray ionization mass spectrometry. Journal of Mass Spectrometry 2003, 38 (5), 491-501.

39.Kitova, E. N.; El-Hawiet, A.; Schnier, P. D.; Klassen, J. S., Reliable Determinations of Protein-Ligand Interactions by Direct ESI-MS Measurements. Are We There Yet? Journal of The American Society for Mass Spectrometry 2012, 23 (3), 431-441.

40.Hopper, J. T. S.; Robinson, C. V., Mass Spectrometry Quantifies Protein Interactions-From Molecular Chaperones to Membrane Porins. Angewandte Chemie International Edition 2014, 53 (51), 14002-14015.

41.Eschweiler, J. D.; Kerr, R.; Rabuck-Gibbons, J.; Ruotolo, B. T., Sizing Up Protein-Ligand Complexes: The Rise of Structural Mass Spectrometry Approaches in the Pharmaceutical Sciences. Annual Review of Analytical Chemistry 2017, 10 (1), 25-44.

42.Robinson, C. V., From molecular chaperones to membrane motors: through the lens of a mass spectrometrist. Biochemical Society Transactions 2017, 45 (1), 251.

43.Light-Wahl, K. J.; Springer, D. L.; Winger, B. E.; Edmonds, C. G.; Camp, D. G.; Thrall, B. D.; Smith, R. D., Observation of a small oligonucleotide duplex by electrospray ionization mass spectrometry. Journal of the American Chemical Society 1993, 115 (2), 803-804. 
44.Brodbelt, J. S., Evaluation of DNA/Ligand Interactions by Electrospray Ionization Mass Spectrometry. Annual Review of Analytical Chemistry 2010, 3 (1), 67-87.

45.Lecours, M. J.; Marchand, A.; Anwar, A.; Guetta, C.; Hopkins, W. S.; Gabelica, V., What stoichiometries determined by mass spectrometry reveal about the ligand binding mode to G-quadruplex nucleic acids. Biochimica et Biophysica Acta (BBA) - General Subjects 2017, 1861 (5), 1353-1361.

46. Marchand, A.; Gabelica, V., Folding and misfolding pathways of G-quadruplex DNA. Nucleic Acids Research 2016, 44 (22), 10999-11012.

47.Porrini, M.; Rosu, F.; Rabin, C.; Darré, L.; Gómez, H.; Orozco, M.; Gabelica, V., Compaction of Duplex Nucleic Acids upon Native Electrospray Mass Spectrometry. ACS Central Science 2017, 3 (5), 454-461.

48.Gabelica, V.; De Pauw, E.; Rosu, F., Interaction between antitumor drugs and a double-stranded oligonucleotide studied by electrospray ionization mass spectrometry. Journal of Mass Spectrometry 1999, 34 (12), 1328-1337.

49.Cassiday, L. A.; Lebruska, L. L.; Benson, L. M.; Naylor, S.; Owen, W. G.; Maher, L. J., Binding Stoichiometry of an RNA Aptamer and Its Transcription Factor Target. Analytical Biochemistry 2002, 306 (2), 290-297.

50.Keller, K. M.; Breeden, M. M.; Zhang, J.; Ellington, A. D.; Brodbelt, J. S., Electrospray ionization of nucleic acid aptamer/small molecule complexes for screening aptamer selectivity. Journal of Mass Spectrometry 2005, 40 (10), 1327-1337.

51.Guo, X.; Liu, Z.; Liu, S.; Bentzley, C. M.; Bruist, M. F., Structural Features of the I-Argininamide-Binding DNA Aptamer Studied with ESI-FTMS. Analytical Chemistry 2006, 78 (20), 7259-7266.

52.Sperry, J. B.; Wilcox, J. M.; Gross, M. L., Strong anion exchange for studying protein-dna interactions by H/D exchange mass spectrometry. Journal of the American Society for Mass Spectrometry 2008, 19 (6), 887-890.

53.Ruigrok, V. J. B.; van Duijn, E.; Barendregt, A.; Dyer, K.; Tainer, J. A.; Stoltenburg, R.; Strehlitz, B.; Levisson, M.; Smidt, H.; van der Oost, J., Kinetic and Stoichiometric Characterisation of Streptavidin-Binding Aptamers. ChemBioChem 2012, 13 (6), 829-836.

54.Trelle, M. B.; Dupont, D. M.; Madsen, J. B.; Andreasen, P. A.; Jørgensen, T. J. D., Dissecting the Effect of RNA Aptamer Binding on the Dynamics of Plasminogen Activator Inhibitor 1 Using Hydrogen/Deuterium Exchange Mass Spectrometry. ACS Chemical Biology 2014, 9 (1), 174-182.

55.Zavyalova, E.; Tagiltsev, G.; Reshetnikov, R.; Arutyunyan, A.; Kopylov, A., Cation Coordination Alters the Conformation of a Thrombin-Binding G-Quadruplex DNA Aptamer That Affects Inhibition of Thrombin. Nucleic Acid Therapeutics 2016, 26 (5), 299-308.

56.Padlan, C. S.; Malashkevich, V. N.; Almo, S. C.; Levy, M.; Brenowitz, M.; Girvin, M. E., An RNA aptamer possessing a novel monovalent cation-mediated fold inhibits lysozyme catalysis by inhibiting the binding of long natural substrates. RNA 2014, 20 (4), 447-461.

57.Phillips, K.; Dauter, Z.; Murchie, A. I. H.; Lilley, D. M. J.; Luisi, B., The crystal structure of a parallel-stranded

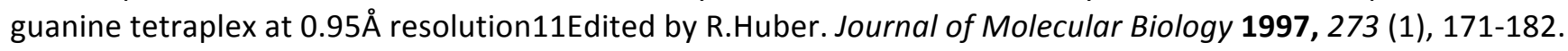

58.Young, M. A.; Jayaram, B.; Beveridge, D. L., Intrusion of Counterions into the Spine of Hydration in the Minor Groove of B-DNA: Fractional Occupancy of Electronegative Pockets. Journal of the American Chemical Society $1997,119(1), 59-69$.

59.Hud, N. V.; Sklenár, V.; Feigon, J., Localization of ammonium ions in the minor groove of DNA duplexes in solution and the origin of DNA A-tract bending11Edited by I. Tinoco. Journal of Molecular Biology 1999, 286 (3), 651-660.

60.Rosu, F.; Gabelica, V.; Poncelet, H.; De Pauw, E., Tetramolecular G-quadruplex formation pathways studied by electrospray mass spectrometry. Nucleic Acids Research 2010, 38 (15), 5217-5225.

61.Ferreira, R.; Marchand, A.; Gabelica, V., Mass spectrometry and ion mobility spectrometry of G-quadruplexes. A study of solvent effects on dimer formation and structural transitions in the telomeric DNA sequence d(TAGGGTTAGGGT). Methods 2012, 57 (1), 56-63.

62.Stojanovic, M. N.; de Prada, P.; Landry, D. W., Aptamer-Based Folding Fluorescent Sensor for Cocaine. Journal of the American Chemical Society 2001, 123 (21), 4928-4931.

63.Stojanović, M. N.; Green, E. G.; Semova, S.; Nikić, D. B.; Landry, D. W., Cross-Reactive Arrays Based on ThreeWay Junctions. Journal of the American Chemical Society 2003, 125 (20), 6085-6089.

64.Pei, R.; Shen, A.; Olah, M. J.; Stefanovic, D.; Worgall, T.; Stojanovic, M. N., High-resolution cross-reactive array for alkaloids. Chemical Communications 2009, (22), 3193-3195. 
65.Reinstein, O.; Yoo, M.; Han, C.; Palmo, T.; Beckham, S. A.; Wilce, M. C. J.; Johnson, P. E., Quinine Binding by the Cocaine-Binding Aptamer. Thermodynamic and Hydrodynamic Analysis of High-Affinity Binding of an Off-Target Ligand. Biochemistry 2013, 52 (48), 8652-8662.

66.Lin, C. H.; Patei, D. J., Structural basis of DNA folding and recognition in an AMP-DNA aptamer complex: distinct architectures but common recognition motifs for DNA and RNA aptamers complexed to AMP. Chemistry \& Biology 1997, 4 (11), 817-832.

67.Robertson, S. A.; Harada, K.; Frankel, A. D.; Wemmer, D. E., Structure Determination and Binding Kinetics of a DNA Aptamer-Argininamide Complex. Biochemistry 2000, 39 (5), 946-954.

68. Neves, M. A. D.; Reinstein, O.; Johnson, P. E., Defining a Stem Length-Dependent Binding Mechanism for the Cocaine-Binding Aptamer. A Combined NMR and Calorimetry Study. Biochemistry 2010, 49 (39), 8478-8487.

69.Sharawy, M.; Consta, S., How do non-covalent complexes dissociate in droplets? A case study of the desolvation of dsDNA from a charged aqueous nanodrop. Physical Chemistry Chemical Physics 2015, 17 (38), 25550-25562.

70.Sun, J.; Kitova, E. N.; Sun, N.; Klassen, J. S., Method for Identifying Nonspecific Protein-Protein Interactions in Nanoelectrospray lonization Mass Spectrometry. Analytical Chemistry 2007, 79 (21), 8301-8311.

71.Sun, J.; Kitova, E. N.; Wang, W.; Klassen, J. S., Method for Distinguishing Specific from Nonspecific Protein-Ligand Complexes in Nanoelectrospray Ionization Mass Spectrometry. Analytical Chemistry 2006, 78 (9), 3010-3018.

72.Gavriilidou, A. F. M.; Gülbakan, B.; Zenobi, R., Influence of Ammonium Acetate Concentration on ReceptorLigand Binding Affinities Measured by Native Nano ESI-MS: A Systematic Study. Analytical Chemistry 2015, 87 (20), 10378-10384.

73.Xia, T.; Yuan, J.; Fang, X., Conformational Dynamics of an ATP-Binding DNA Aptamer: A Single-Molecule Study. The Journal of Physical Chemistry B 2013, 117 (48), 14994-15003.

74.Ying, Y.-L.; Wang, H.-Y.; Sutherland, T. C.; Long, Y.-T., Monitoring of an ATP-Binding Aptamer and its Conformational Changes Using an $\alpha$-Hemolysin Nanopore. Small 2011, 7 (1), 87-94.

75. Harada, K.; Frankel, A. D., Identification of two novel arginine binding DNAs. The EMBO Journal 1995, 14 (23), 5798-5811.

76.Bishop, G. R.; Ren, J.; Polander, B. C.; Jeanfreau, B. D.; Trent, J. O.; Chaires, J. B., Energetic basis of molecular recognition in a DNA aptamer. Biophysical Chemistry 2007, 126 (1-3), 165-175.

77.Lin, P.-H.; Tsai, C.-W.; Wu, J. W.; Ruaan, R.-C.; Chen, W.-Y., Molecular dynamics simulation of the induced-fit binding process of DNA aptamer and L-argininamide. Biotechnology Journal 2012, 7 (11), 1367-1375.

78.Albada, H. B.; Golub, E.; Willner, I., Computational docking simulations of a DNA-aptamer for argininamide and related ligands. Journal of Computer-Aided Molecular Design 2015, 29 (7), 643-654.

79.Cekan, P.; Jonsson, E. Ö.; Sigurdsson, S. T., Folding of the cocaine aptamer studied by EPR and fluorescence spectroscopies using the bifunctional spectroscopic probe Ç. Nucleic Acids Research 2009, 37 (12), 3990-3995.

80.Sachan, A.; Ilgu, M.; Kempema, A.; Kraus, G. A.; Nilsen-Hamilton, M., Specificity and Ligand Affinities of the Cocaine Aptamer: Impact of Structural Features and Physiological NaCl. Analytical Chemistry 2016, 88 (15), 77157723.

81.Neves, M. A. D.; Shoara, A. A.; Reinstein, O.; Abbasi Borhani, O.; Martin, T. R.; Johnson, P. E., Optimizing Stem Length To Improve Ligand Selectivity in a Structure-Switching Cocaine-Binding Aptamer. ACS Sensors 2017, 2 (10), 1539-1545.

82.Slavkovic, S.; Altunisik, M.; Reinstein, O.; Johnson, P. E., Structure-affinity relationship of the cocaine-binding aptamer with quinine derivatives. Bioorganic \& Medicinal Chemistry 2015, 23 (10), 2593-2597.

83.Reinstein, O.; Neves, M. A. D.; Saad, M.; Boodram, S. N.; Lombardo, S.; Beckham, S. A.; Brouwer, J.; Audette, G. F.; Groves, P.; Wilce, M. C. J.; Johnson, P. E., Engineering a Structure Switching Mechanism into a Steroid-Binding Aptamer and Hydrodynamic Analysis of the Ligand Binding Mechanism. Biochemistry 2011, 50 (43), 9368-9376.

84.Neves, M. A. D.; Reinstein, O.; Saad, M.; Johnson, P. E., Defining the secondary structural requirements of a cocaine-binding aptamer by a thermodynamic and mutation study. Biophysical Chemistry 2010, 153 (1), 9-16.

85.Paul, F.; Weikl, T. R., How to Distinguish Conformational Selection and Induced Fit Based on Chemical Relaxation Rates. PLoS Computational Biology 2016, 12 (9), e1005067.

86.Chakraborty, P.; Di Cera, E., Induced Fit Is a Special Case of Conformational Selection. Biochemistry 2017, 56 (22), 2853-2859. 
87.Morando, M. A.; Saladino, G.; D’Amelio, N.; Pucheta-Martinez, E.; Lovera, S.; Lelli, M.; López-Méndez, B.; Marenchino, M.; Campos-Olivas, R.; Gervasio, F. L., Conformational Selection and Induced Fit Mechanisms in the Binding of an Anticancer Drug to the c-Src Kinase. Scientific Reports 2016, 6, 24439.

88. Huizenga, D. E.; Szostak, J. W., A DNA Aptamer That Binds Adenosine and ATP. Biochemistry 1995, 34 (2), 656665.

89.Yangyuoru, P. M.; Dhakal, S.; Yu, Z.; Koirala, D.; Mwongela, S. M.; Mao, H., Single-Molecule Measurements of the Binding between Small Molecules and DNA Aptamers. Analytical Chemistry 2012, 84 (12), 5298-5303.

90.Zhang, Z.; Oni, O.; Liu, J., New insights into a classic aptamer: binding sites, cooperativity and more sensitive adenosine detection. Nucleic Acids Research 2017, 45 (13), 7593-7601.

91.Vogt, A. D.; Di Cera, E., Conformational Selection or Induced Fit? A Critical Appraisal of the Kinetic Mechanism. Biochemistry 2012, 51 (30), 5894-5902.

92.Vogt, A. D.; Di Cera, E., Conformational Selection Is a Dominant Mechanism of Ligand Binding. Biochemistry 2013, 52 (34), 5723-5729.

93. Michel, D., Conformational selection or induced fit? New insights from old principles. Biochimie 2016, 128 (Supplement C), 48-54.

94.Greives, N.; Zhou, H.-X., Both protein dynamics and ligand concentration can shift the binding mechanism between conformational selection and induced fit. Proceedings of the National Academy of Sciences 2014, 111 (28), 10197-10202.

95.Wolff, P.; Da Veiga, C.; Ennifar, E.; Bec, G.; Guichard, G.; Burnouf, D.; Dumas, P., Native ESI Mass Spectrometry Can Help to Avoid Wrong Interpretations from Isothermal Titration Calorimetry in Difficult Situations. Journal of The American Society for Mass Spectrometry 2017, 28 (2), 347-357. 